\title{
Stiffness and position control of a prosthetic wrist by means of an EMG interface
}

\author{
Shodhan Rao, Raffaella Carloni and Stefano Stramigioli
}

\begin{abstract}
In this paper, we present a novel approach for decoding electromyographic signals from an amputee and for interfacing them with a prosthetic wrist. The model for the interface makes use of electromyographic signals from electrodes placed in agonistic and antagonistic sides of the forearm. The model decodes these signals in order to control both the position and the stiffness of the wrist.
\end{abstract}

\section{INTRODUCTION}

In recent years, the use of biological signals for controlling artificial prostheses has received great attention and research in this direction is of high impact for people who have undergone amputation of their limbs.

Peripheral nervous system interfaces are used for obtaining biological signals for the control of prosthetic limbs [1]. These interfaces are usually implanted at specific points, which are proximal to the amputated section. The signals from these interfaces are then connected to the prosthetic device by means of a control system, which usually consists of a signal amplifier, a filter and a signal processing unit.

In order to perform different activities like grasping and manipulation, humans voluntarily and skillfully steer the activation levels of the agonistic and antagonistic muscles. The difference between the contractions of these two muscles generates the torque required to rotate a joint. If both agonistic and antagonistic muscles are simultaneously activated, the joint stiffness increases. For example, when we clench our fists, we voluntarily activate opposing muscles in the forearm, which leads to a change in stiffness of the arm.

The present methods of control of prosthetic hands/fingers by means of electromyographic (EMG) signals involve translating these signals into the desired positions [2], [3], [4]. This methodology is far from how a human hand is steered by properly adapting the stiffness of the various muscles in order to optimize it with respect to different tasks, like grasping and manipulation, and to different environments.

In this paper, we present a model that receives as input, efferent signals from EMG interfaces placed in the proximity of the amputated section and gives as outputs both the position and the stiffness of a prosthetic wrist. This model is based on the results of experiments conducted on a healthy human subject, wherein he was asked to flex and extend his wrist with varying levels of stiffness, accomplished by artificial resistance, and EMG signals were recorded from

This work has been partially funded by IMPACT Research Institute of the University of Twente, as part of the project ARTHAND.

\{s.rao,r.carloni,s.stramigioli\}@utwente.nl, IMPACT Institute, Dept. Electrical Engineering, Faculty of Electrical Engineering, Mathematics and Computer Science, University of Twente, 7500 AE Enschede, The Netherlands. electrodes placed at flexor and extensor side of the forearm. Since our model is based on how a human being varies his stiffness and position by controlling the activation of his muscles, it is intended to lead to the design of prosthetic devices that give a much more natural feel to the user as compared to current electromyographic prosthetic devices.

In this paper, we propose a novel approach to decode the information present in a EMG signal, which will be henceforth referred to as the signal activation. While several feature extraction methods can be used in order to define EMG activation, we specifically use standard deviation of the signal in order to define EMG activation. Based on the observation of the results of the experiments on a healthy human subject, we believe that our approach for defining EMG activation helps to clearly differentiate between free and stiff wrist flexion and extension.

The methods for EMG based control of prosthetic and exoskeleton devices described in [5] and [6], respectively, are also based on EMG data from extensor and flexor muscles. However, the control laws for rotation about the joints in terms of the extensor and flexor EMG data are different from the one that we propose. While our study focusses on stiffness and position control using EMG, in [5] and [6], impedance control using EMG is accomplished. The EMG decoding algorithm for stiffness and position presented in this paper is different from the ones used in these studies.

The paper is organized as follows. In Sec. II, we present a simple wrist model, in which position and stiffness can be controlled independently by means of two actuators. In Sec. III, we describe the experiments conducted on a healthy human subject in order to understand the relation between EMG and position/stiffness of the human wrist. In Sec. IV, we propose a method to compute the activation of a given EMG signal, and we present an algorithm to decode the desired position and stiffness from EMG data. In Sec. V, we show the result of simulation of the wrist model by using our experimental EMG data, and we conclude in Sec. VI.

\section{A VARIABLE STIFFNESS WRIST MODEL}

In this Section, we describe the model of a simple wrist, for which both position and stiffness can be controlled. The model, as depicted in Fig. 1, consists of two actuators $M_{1}$ and $M_{2}$ and two quadratic springs, i.e the governing law for each spring is $F=k s|s|$, where $s$ denotes the extension/compression of the spring, $k$ the elastic constant of each spring and $F$ the force exerted by each spring. The motor $M_{1}$ is rotational and is used to control the output position, i.e. the angle of rotation $\theta$ of the wrist, while $M_{2}$ 


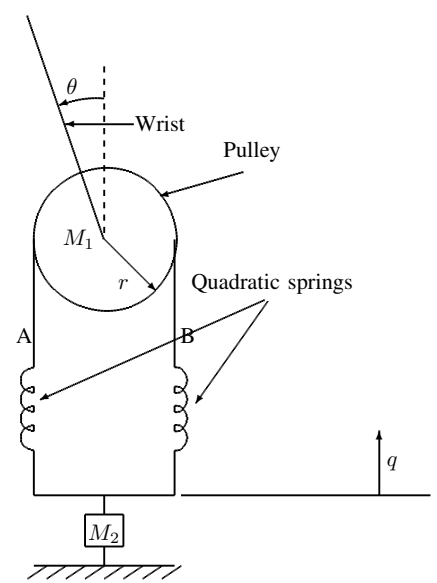

Fig. 1. A variable stiffness wrist model - The rotational motor $M_{1}$ regulates the wrist position, while the linear motor $M_{2}$ is used to change the wrist stiffness.

is a linear motor and is used to control the displacement $q$ and, therefore, the stiffness of the wrist. Assume that $\theta$ is always positive and obeys the inequality $r \theta>|q|$, where $r$ denotes the radius of the pulley. We now show that stiffness of the wrist model is directly proportional to $q$. Observe that since $\theta$ is positive and $r \theta>|q|$, one of the springs gets compressed by length $s_{1}=r \theta-q$, while the other expands by length $s_{2}=r \theta+q$. The forces $F_{1}$ and $F_{2}$ of compression and expansion are given by $F_{1}=k(r \theta-q)^{2}$ and $F_{2}=k(r \theta+q)^{2}$, respectively. The torque applied on the wrist is given by $\tau=\left(F_{2}-F_{1}\right) r=4 k r^{2} q \theta$. The stiffness $K_{f}$ of the wrist is defined as:

$$
K_{f}:=\frac{\partial \tau}{\partial \theta}=4 k r^{2} q
$$

i.e the stiffness is directly proportional to $q$.

Note that while there are several possible mechanical models, in which position and stiffness of a rotational joint can be independently controlled, this model has been chosen for the present study because it closely resembles the model of a prosthetic hand developed in the Control Engineering Group of the University of Twente.

\section{EXPERIMENT DESCRIPTION}

In this Section, we describe the experiments conducted on a healthy human subject at Roessingh, Research and Development (RRD) in Enschede, The Netherlands. The scope of the experiments is to obtain EMG data corresponding to flexion and extension of the wrist at different levels of stiffness. These experiments were fundamental for understanding the relation between the temporal features of the EMG data and the information on position/stiffness of the wrist. The main contribution of this paper is on how to use the obtained relation in order to control a simple wrist in both position and stiffness and, therefore, on how to extract position/stiffness information from EMG data.

We now describe the details of the experiments. Initially, the healthy human subject was endowed with four electrodes in anatomical locations. An electrode was placed on either

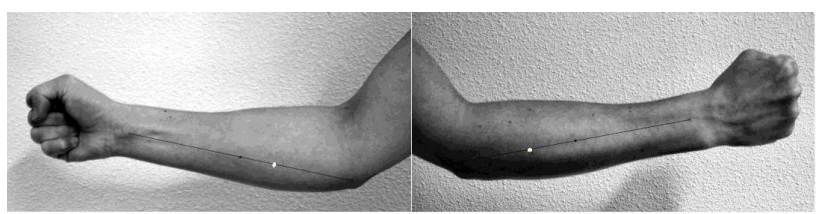

Fig. 2. Experimentas - An electrode is placed on either side of the two dots along the two lines.

side of the two dots along the two lines shown in Fig. 2. Thus effectively, two electrodes were placed on the flexor side and two on the extensor side of the forearm. Moreover, a reference electrode was placed close to the subject's neck.

The human subject was made to sit comfortably on a chair with the forearm placed on a table of adjustable height. Initially the subject was asked to freely flex and extend his wrist for about $15 \mathrm{~s}$ and the corresponding EMG data was recorded through two channels, one corresponding to the medial electrodes (flexor muscle) and the other corresponding to the lateral electrodes (extensor muscle). Henceforth these will be referred to as flexor and extensor EMG data respectively. In $15 \mathrm{~s}$, the subject completed 18 cycles of wrist movement. After a resting period, the subject was asked to extend and flex his wrist in the presence of a resisting force, applied at the subject's palm and recorded by using a hand held dynamometer. This created a different stiffness level of the wrist. During this part of the experiment, by observation of the dynamometer, the resisting force was continuously reduced during the course of half cycle of the wrist movement from a maximum level to zero. This experiment was conducted for $20 \mathrm{~s}$ and consisted of about three flexions and two extensions of the wrist. The EMG data corresponding to this experiment was also recorded.

\section{DECODING EMG DATA}

The information contained in the EMG data, defined as its activation, has to be decoded in order to obtain meaningful signals to be given to a prosthetic device.

The sampling frequency of the EMG data is $1024 \mathrm{~Hz}$. We divide the time-domain of each experiment into small time intervals of approximately $0.1 \mathrm{~s}$, and then compute the mean value of the signal over each of these time intervals. For the $n^{\text {th }}$ time interval, let $f(n)$ denote this mean value. In a few references (see for example [7]), such a measure is considered as the neural activity/energy or information present in the signal. The following observations are made on our experimental EMG data:

- There is a small variation of the mean value of EMG data corresponding to each electrode over the entire time-domain of the experiments and, hence, it provides little information about position and stiffness.

- During every flexion (both free and stiff) of the wrist, there is a significant variation of the flexor EMG data about its mean value, and less variation of extensor EMG data about its mean. Likewise, it is observed that during every extension, there is a significant variation 
of the extensor EMG data about its mean value, and less variation of flexor EMG data about its mean.

- The flexor EMG data corresponding to stiff extension has a larger variation about its mean as compared to the flexor EMG data corresponding to free extension. Likewise, the extensor EMG data corresponding to stiff flexion has a larger variation about its mean as compared to the extensor EMG data corresponding to free flexion.

The above observations prompt us to define activation using the following approach. Since the sampling frequency of our EMG data is $1024 \mathrm{~Hz}$, each time segment of $T=0.1 \mathrm{~s}$ has roughly $m=102$ samples of data. Let $G(i, n)$ denote the EMG value of the $i^{\text {th }}$ sample in the $n^{\text {th }}$ time segment. Then the activation $A(t)$ of the signal at time $t$ is defined as follows:

$$
A(t)=\sqrt{\frac{1}{m} \sum_{i=1}^{m}(G(i, n)-f(n))^{2}}, \quad(n-1) T<t \leq n T
$$

Note that the activation at any time is the standard deviation of the signal for the segmented time interval in which the time instant under consideration lies. Figs. 3 and 4 give the plots of flexor and extensor activations during free and stiff wrist movements, respectively.

Denote the flexor and extensor activations with $F, E$ respectively, where $F, E: \mathbb{R}_{+} \rightarrow \mathbb{R}_{+}$and $\mathbb{R}_{+}$is the set of positive real numbers. Define $P=F-E$. From the data corresponding to graphs in Figs. 3 and 4, the following inferences can be made:

- During every cycle of the wrist movement (both free and stiff) consisting of a flexion and an extension, the variation of $P$ with time is cyclic in such a way that during flexion, its value goes from approximately 0 to a maximum of about 300 and then falls back to 0 , and during extension, its value goes to a minimum of about -300 and then rises back to approximately 0 . Hence $P$ can be used as a measure of wrist position.

- The flexor activation during free extension and extensor activation during free flexion are mostly less than a threshold $G_{T}$, i.e. 22 in the specific experiment. In other words, if $G_{T}$ is considered as the value of threshold activation for this experiment, then during free flexion, only the flexor is mostly activated, while during free extension, only the extensor is mostly activated.

- The flexor activation during stiff extension and extensor activation during stiff flexion vary between 0 and 100 in the specific experiments. Again if we consider $G_{T}=22$, this implies that stiff movement involves the activation of both the flexor and the extensor, such that flexor activation is higher during flexion and extensor activation is higher during extension.

We make use of the above analysis to devise a methodology that takes as input the flexor and extensor activations, computes the desired position $\theta_{d}$ and stiffness $K_{f d}$ of the prosthetic wrist by using these activations and controls the position and stiffness of the prosthetic wrist model described

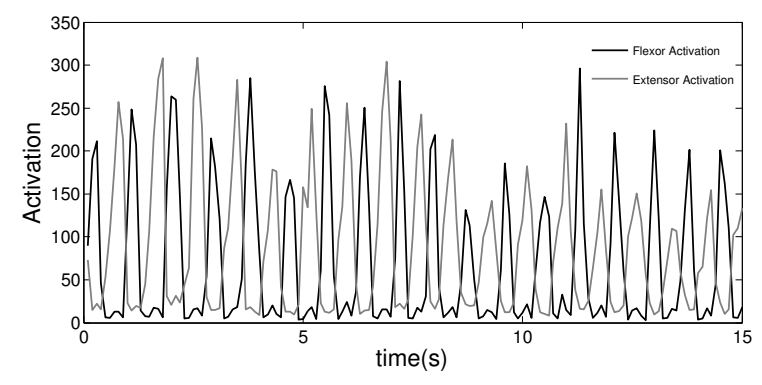

Fig. 3. Flexor and extensor activation during free wrist movement described by the black and grey curves, respectively.

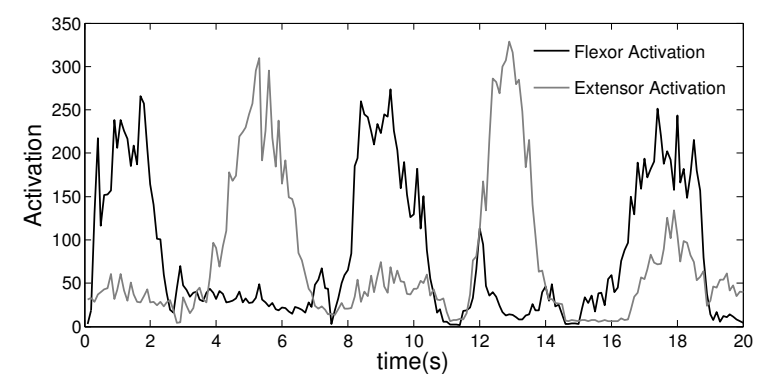

Fig. 4. Flexor and extensor activation during stiff wrist movement described by the black and grey curves, respectively.

in Sec. II by means of PID controllers. Define $q_{d}:=-\frac{K_{f d}}{4 k r^{2}}$, where $k$ and $r$ denote the elastic constant of the quadratic springs and the radius of the pulley in the wrist model described in Sec. II. We propose that the desired angle $\theta_{d}=a_{1}(F-E)+a_{2}$ where $a_{1}$ and $a_{2}$ are constants chosen in such a way that $\theta_{d}$ is always positive and $r \theta_{d}>\left|q_{d}\right|$. Let $a_{3}$ denote the minimum value of the desired stiffness $K_{f d}$. If either flexor or extensor activation is less than the threshold $G_{T}$, then we propose that $K_{f d}=a_{3}$, else we propose that $K_{f d}$ depends linearly on the minimum of flexor and extensor activations. Algorithm 1 based on these ideas can be used for the computation of the desired position $\left(\theta_{d}\right)$ and stiffness $\left(K_{f d}\right)$, given the flexor and extensor activations. The value of $G_{T}$ is determined by observation of the EMG data and it depends on both the human subject and also the position of the electrodes. With respect to Algorithm 1, $a_{4}$ is a constant.

Algorithm 1 Input: Flexor activation $\left(F: \mathbb{R}_{+} \rightarrow \mathbb{R}_{+}\right)$and extensor activation $\left(E: \mathbb{R}_{+} \rightarrow \mathbb{R}_{+}\right)$corresponding to wrist movement.

Output: Desired position $\theta_{d}$ and stiffness $K_{f d}$ of a prosthetic wrist.

1. Define $\theta_{d}=a_{1}(F-E)+a_{2}$.

2. For $t$ within the time domain of the wrist movement, If $F(t)<G_{T}$ or $E(t)<G_{T}$, then $K_{f d}=a_{3}$ Elseif $F(t)<E(t)$, then

$$
K_{f d}=a_{3}+\left(F(t)-G_{T}\right) a_{4}
$$

Else $K_{f d}=a_{3}+\left(E(t)-G_{T}\right) a_{4}$

3. Output $\theta_{d}, K_{f d}$. 

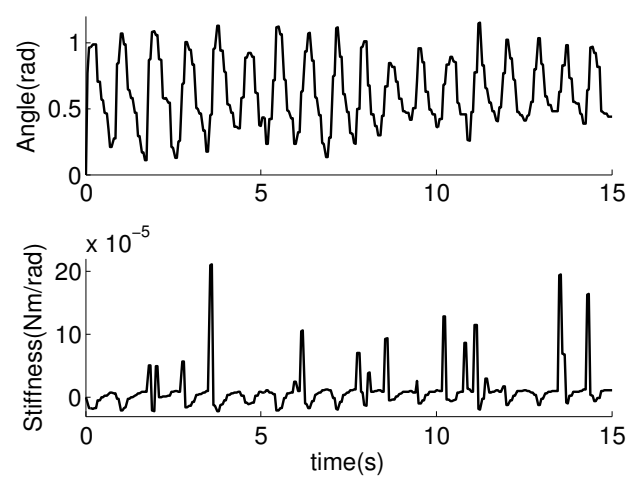

Fig. 5. Angular position and stiffness vs time for free movement.

\section{SIMULATIONS}

We simulate the simple wrist model described in Sec. II with PID controllers for motors $M_{1}$ and $M_{2}$. The desired values of position and stiffness for the PID controllers are obtained using Algorithm 1, with the flexor and extensor activations obtained from our experiments as inputs. The following parameter values are considered for the model: $r=0.01 \mathrm{~m}, k=180 \mathrm{~N} / \mathrm{m}, a_{1}=0.0018, a_{2}=0.65$, $a_{3}=a_{4}=0.0001$, moment of inertia $(I)$ of the link about its rotation axis given by $I=1.25 \times 10^{-4} \mathrm{kgm}^{2}$. The simulation is carried out using 20-sim software [8].

Fig. 5 and 6 show the variation of angular position and stiffness of the wrist with respect to time for the free and stiff movements respectively obtained through simulation. From the graphs, it can be observed that angular position corresponding to both stiff and free movements varies between 0 and $1.2 \mathrm{rad}$. For stiff movement, the wrist stiffness remains high for a longer period of time as compared to free movement, in case of which there are small time instants (about $0.1 \mathrm{~s}$ long), when the magnitude of stiffness suddenly increases. This can be explained as being caused mostly when the wrist changes its direction of rotation, because the force opposing the rotation is high at such times. While for stiff movement, the maximum stiffness obtained through our simulation is $0.0008 \mathrm{Nm} / \mathrm{rad}$, for free movement this value is equal to $0.00021 \mathrm{Nm} / \mathrm{rad}$. It has been verified that $r \theta>|q|$, during the time-domain of the simulation.

\section{CONCLUSION}

We conclude that simultaneous activation of both flexor and extensor muscles leads to stiff movement of the wrist, while activation of only one of the two muscles implies free movement of the wrist. Using this principle, we have designed an algorithm that computes the desired position and stiffness of a prosthetic wrist model. These desired values can be used to actuate the motors of a simple wrist model described in Sec. II in order to control its position and stiffness. Our study can be used to design a prosthetic hand for amputees based on experimental studies on their healthy hand in order to replace the inexistent hand. Algorithm 1
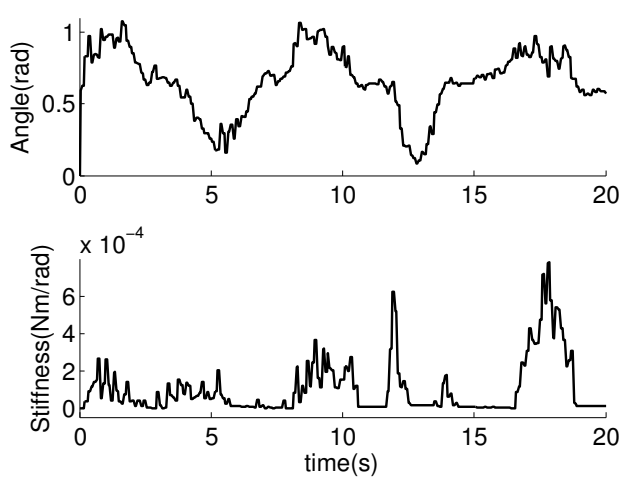

Fig. 6. Angular position and stiffness vs time for stiff movement.

does not provide precise decoding of the actual position and stiffness of the human wrist from experimental EMG data. We believe that, once a prosthetic hand designed on the basis of experimental data from the healthy hand of an amputee is used to replace the inexistent hand, he/she can learn to control its stiffness and position by properly activating specific muscles over an initial training period when the amputee becomes compatible with the use of the prosthetic device.

A future direction of research is to perform EMG-based experiments on healthy humans with precise measurements of angular position and stiffness in order to obtain precise decoding algorithms. Another direction is to experimentally analyze the difference between the two hands in terms of position and stiffness control as described in this paper. This will help to understand the effectiveness of using the healthy hand of an amputee in order to design prosthetic control.

\section{ACKNOWLEDGMENTS}

We are grateful to Dr. Laura Kallenberg of Roessingh Research and Development in Enschede, The Netherlands, for giving us the opportunity and the necessary help to conduct the experiments described in this paper.

\section{REFERENCES}

[1] X. Navarro, T.B. Krueger, N. Lago, S. Micera, T. Stieglitz and P. Dario, "A critical review of interfaces with the peripheral nervous system for the control of neuroprostheses and hybrid bionic systems", Jour. Peripheral Nervous System, vol. 10, n. 3, pp. 229-258, 2005.

[2] http://www.ottobockus.com

[3] http://www.touchbionics.com/

[4] http://spectrum.ieee.org/tag/prosthetic arm

[5] S. Lee, Y. Sankai, "Power assist control for walking aid with HAL-3 based on EMG and Impedance adjustment around knee joint", Int. Conf. on Intelligent Robots and Systems, pp. 1499-1504, 2002.

[6] T. Tsuji, O. Fukuda, H. Shigeyoshi and M. Kaneko, "Bio-mimetic impedance control of an EMG-controlled prosthetic hand", Int. Conf. on Intelligent Robots and Systems, pp. 377-382, 2000.

[7] J.L. Feber, E.V. Asselt and R.V. Mastrigt, "Neurophysiological modeling of voiding in rats: bladder pressure and postganglionic bladder nerve activity", American Jour. Physiology - Regulatory, Integrative and Comparative Physiology, vol. 272, pp. 413-421, 1997.

[8] Controllab Products B.V., “20-sim”, http://www.20sim.com, 2010. 